\title{
Determination of buffer capacity by means of an ISFET-based coulometric sensor-actuator system with a gate-covering porous actuator
}

\author{
J. Luo, W. Olthuis and P. Bergveld \\ MESA Research Institute, University of Twente, PO Bax 217, 7500 AE Enschede (Netherlands) \\ M. Bos and W.E. van der Linden \\ Department of Chemical Technology, University of Twente, PO Box 217, 7500 AE Enschede (Netherlands)
}

(Received March 10, 1993; accepted July 10, 1993)

\begin{abstract}
In this paper we propose a dynamic way to measure the buffer capacity of an electrolyte by means of an ISFETbased coulometric sensor-actuator system whose gate is covered with a porous actuator. A theoretical model for this measurement is presented. Experiments are carried out in nitric and acetic acid as well as in phosphoric acid solutions. A fairly good agreement has been found between the theoretical calculations and the experimental results.
\end{abstract}

Introduction

The ISFET as a $\mathrm{pH}$ sensor has shown some unique advantages over the traditional ion-selective electrodes in somc spccific ficlds, such as clinical applications or in vivo monitoring, although it still faces the problem of drift. The integration of a coulometric actuator with an ISFET has led to applications where the drift problem is circumvented. This coulometric sensor-actuator system has successfully been applied to acid-base titrations $[1,2]$ and the construction of a novel carbon dioxide sensor [3]. So far, this coulometric system can measure both the $\mathrm{pH}$ and the concentration of an analyte simultaneously. The construction of a pH-static enzyme sensor employing this system shows the ability of local $\mathrm{pH}$ control in the vicinity of the gate of an ISFET [4]. Yet a limitation arising from a delay in response has restricted the applicability of this system. The semiquantitative description of this delay time demonstrated a dependence of the delay on the distance between the sensor and the actuator as well as on the mobility of the species [5]. In order to circumvent this problem, a newly designed coulometric sensor-actuator system that employs a porous gold actuator closely over the gate of the ISFET has been proposed [6]. The basic components of the system are shown in Fig. 1. The experimental results have shown a considerably reduced delay in system response [6, 7]. A theoretical study has shown that the reduced delay time should be ascribed

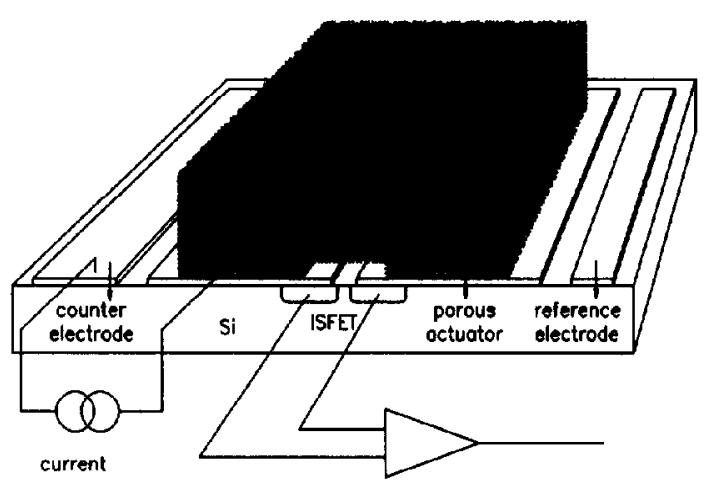

Fig. 1. Schematic representation of the basic components of the coulometric sensor-actuator system based on an ISFET with gate-covering porous actuator.

not only to the largely shortened distance between the sensor and actuator, but also to the diffusion-limiting barrier of the porous actuator [7]. For application to acid-base titration, if the thickness of the actuator is large in comparison to the thickness of the diffusion layer of the species, the time to reach the equivalence point has been found to be approximately linearly proportional to the concentration of the titrated species [6].

The new system has a highly porous actuator closely covering the ISFET. A thick porous layer is in itself a small reaction chamber around the gate of the ISFET. The $\mathrm{pH}$ sensing and changing are both taking place 
inside this chamber. If a small perturbation of titrant is coulometrically generated by applying an alternating current to the porous actuator, the associated $\mathrm{pH}$ change inside the porous actuator will be a function of the buffer capacity of the analyte and can be rapidly detected by the ISFET. This provides a possibility for the new coulometric sensor-actuator system to determine the buffer capacity.

In this paper, we propose a new approach that utilizes this alternating generation of protons and hydroxyl ions for a dynamic determination of the buffer capacity of an analyte.

\section{Principle of operation}

The titrant coulometrically generated at the actuator by electrolysis of water can be either protons or hydroxyl ions, depending on the direction of the applied current. The typical reactions at the electrodes are

anode: $\quad 2 \mathrm{H}_{2} \mathrm{O} \longrightarrow 4 \mathrm{H}^{+}+4 e^{-}+\mathrm{O}_{2}$

cathode: $2 \mathrm{H}_{2} \mathrm{O}+2 e^{-} \longrightarrow 2 \mathrm{OH}^{-}+\mathrm{H}_{2}$

If a titration is carried out, a constant generation of titrant is required until the depletion of the titrated species. This depletion can be easily detected as the end-point by the sensor. Note that the applied current is usually constant in this case. For a determination of the buffer capacity, one needs to differentiate the full titration curve in such a way. A more convenient way proposed here is the application of a programmed current to measure the buffer capacity directly.

Buffer capacity is by definition the reciprocal of the slope of the titration curve. Besides the conventional way that uses a titration, the buffer capacity can also be measured by applying a small perturbation of titrant at different $\mathrm{pH}$ values. This is illustrated in Fig. 2 . The solid curved line shown in this Figure is the titration curve of an acid. The abscissa represents the equivalents of added base (left from zero) and acid (right from zero) to the original species. If a small amount of titrant is added in an alternating manner as shown in this Figure, the resulting $\mathrm{pH}$ changes will depend on the buffer capacity of the solution, i.e., the reciprocal of the slope of the titration curve at the $\mathrm{pH}$ concerned. The perturbation of titrant can be coulometrically generated and the modulated $\mathrm{pH}$ can be detected by an ISFET. Both of these can be achieved by an ISFETbased coulometric sensor-actuator system with a gatecovering porous actuator. This operation of the system provides a dynamic way of measuring the buffer capacity. For reasons of simplicity, sinusoidal coulometry will be adopted since it facilitates the mathematical treatment. The response of the system and the determination of the buffer capacity will be dealt with in the next section.

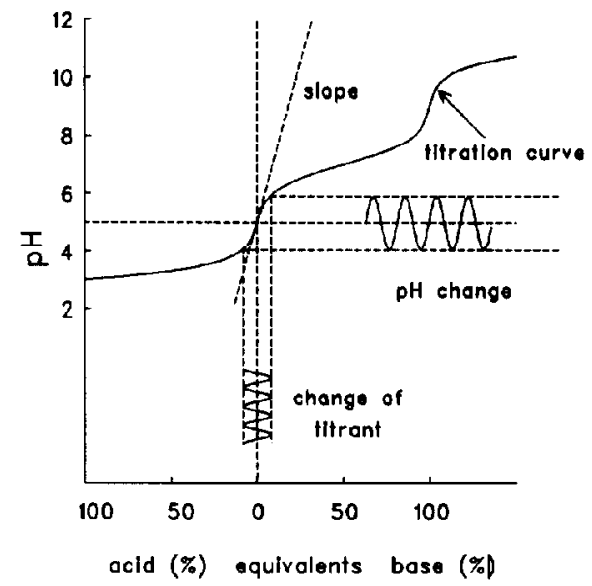

Fig. 2. Illustration of the buffer capacity measurement by means of an applied sinusoidal perturbation of titrant.
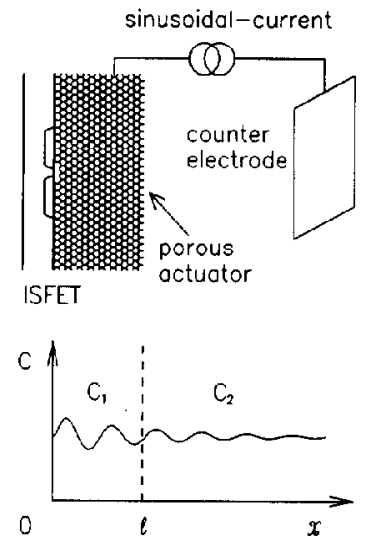

Fig. 3. Illustration of the concentration profiles inside and outside the porous actuator for $t>0$.

\section{Mathematical description}

\section{System response to a sinusoidal current actuation}

For the modelling of the ISFET response to a sinusoidal applied actuator current, some assumptions similar to those for a coulometric titration have to be made [7]. These assumptions are:

(1) The diffusion is considered to be one dimensional, which is reasonable because the geometric area of the actuator is much larger than its thickness. The concentration profiles of the species are divided into two parts as shown in Fig. 3, where $x$ is the distance from the gate to the bulk, perpendicular to the ISFET, and $l$ is the thickness of the porous actuator. $C_{1}(x, t)$ and $C_{2}(x, t)$ represent the concentration profiles of the species inside and outside the porous actuator, respectively.

(2) The diffusion coefficients of the species inside the porous actuator are considered to be the same as 
in the bulk solution because of the relatively large pore size of the actuator.

(3) The porosity of the porous actuator is assumed to be homogeneous, and the applied current is assumed to be uniformly distributed over the whole active surface of the porous actuator. As a consequence of this assumption, the titrant is then considered to be uniformly generated inside the porous actuator. The volume occupied by the gold itself is ignored because of the reasonably high porosity of the actuator.

(4) Since the $\mathrm{pH}$ is measured by an ISFET, it is no longer necessary to determine the current density at the electrode for the calculation of the electrode potential. When a current is applied to the actuator, the titrant generated in the internal volume of the actuator can be considered as a homogeneous titrant source.

With the assumptions made above, the derivation can proceed. First, a unit $\rho_{b}$ defined as the titrant generated per unit time and per unit volume, will be introduced [7] for the description of the titrant source.

For an applied sinusoidal current, the generation of titrant will exhibit a certain initial phase shift, $\theta$, because of the charge transfer and the mass transport from the actuator surface into the pores. When this initial phase shift is taken into account, the applied sinusoidal current can be defined as

$i=I \cos (\omega t-\theta)$

where $I$ is the maximum value of the amplitude and $\omega$ is the angular frequency of the applied current, respectively. Then, the titrant production rate per unit volume, $\rho_{f}$, as has just been defined, is [7]

$\rho_{\mathrm{f}}=\frac{I_{\mathrm{f}} \cos [(\omega t-\theta)+\theta]}{F V_{\text {act }}}=\frac{I_{\mathrm{f}} \cos \omega t}{F S l}$ where $I_{f}$ is the faradaic portion of the amplitude of the applied current $I$ and $F$ the Faraday constant, $V_{\text {act }}$ is the total volume of the porous actuator, $S$ and $l$ are the geometric area and thickness of the porous actuator, respectively. Here the subscript $f$ is used to denote the corresponding faradaic quantity, because only the faradaic current takes part in the electrode reaction.

The equations governing the mass transport of the electroactive species inside and outside the porous actuator are expressed as [7]

$$
\begin{aligned}
\frac{\partial C_{1}(x, t)}{\partial t} & =D \frac{\partial^{2} C_{1}(x, t)}{\partial x^{2}}+\rho_{\mathrm{f}} \\
& =D \frac{\partial^{2} C_{1}(x, t)}{\partial x^{2}}+K \cos \omega t \quad 0<x<l \\
\frac{\partial C_{2}(x, t)}{\partial t} & =D \frac{\partial^{2} C_{2}(x, t)}{\partial x^{2}} \quad l<x<\infty
\end{aligned}
$$

where $D$ is the diffusion coefficient of the electroactive species and $K=l_{\mathrm{f}} / F S l$. The initial and boundary conditions are

$$
\begin{aligned}
& t=0, C_{1}(x, 0)=C_{2}(x, 0)=C_{0} \\
& x=0, \frac{\partial C_{1}(0, t)}{\partial x}=0 \\
& x=l,\left\{\begin{array}{l}
\frac{\partial C_{1}(l, t)}{\partial x}=\frac{\partial C_{2}(l, t)}{\partial x} \\
C_{1}(l, t)=C_{2}(l, t)
\end{array}\right. \\
& x \rightarrow \infty, C_{2}(x, t)=C_{0}
\end{aligned}
$$

where $C_{0}$ is the initial bulk concentration of the species.

The solutions for eqns. (4) and (5) are [8]

$$
\begin{aligned}
C_{1}(x, t)= & C_{0}+\frac{K}{\omega} \sin \omega t-\frac{K}{2 \omega}\left\{\exp \left[-\left(\frac{\omega}{2 D}\right)^{1 / 2}(l+x)\right] \sin \left[\omega t-\left(\frac{\omega}{2 D}\right)^{1 / 2}(l+x)\right]\right. \\
& +\exp \left[-\left(\frac{\omega}{2 D}\right)^{1 / 2}(l-x)\right] \sin \left[\omega t-\left(\frac{\omega}{2 D}\right)^{1 / 2}(l-x)\right] \\
& \left.+\frac{1}{\pi} \int_{0}^{\infty} \frac{\omega D}{\omega^{2}+(D Z)^{2}} \exp (-D t Z)\left[\sin (l+x)(Z)^{1 / 2}+\sin (l-x)(Z)^{1 / 2}\right] \mathrm{d} Z\right\} \\
C_{2}(x, t)= & C_{0}+\frac{K}{2 \omega}\left\{\exp \left[-\left(\frac{\omega}{2 D}\right)^{1 / 2}(x-l)\right] \sin \left[\omega t-\left(\frac{\omega}{2 D}\right)^{1 / 2}(x-l)\right]+\exp \left[-\left(\frac{\omega}{2 D}\right)^{1 / 2}(x+l)\right] \sin \left[\omega t-\left(\frac{\omega}{2 D}\right)^{1 / 2}(x+l)\right]\right. \\
& \left.+\frac{1}{\pi} \int_{0}^{\infty} \frac{\omega D}{\omega^{2}+(D Z)^{2}} \exp (-D t Z)\left[\sin (x-l)(Z)^{1 / 2}+\sin (x+l)(Z)^{1 / 2}\right] \mathrm{dZ}\right\}
\end{aligned}
$$

The infinite integral part in the right-hand side of eqns. (10) and (11) represents the transient part of the response, which can be ignored under steady-state conditions. Since the detection is carried out by the gate of 
the ISFET, which is very closely located to the edge of the actuator $(x=0)$, only the solution $C_{1}(0, t)$ is of interest. Because only the steady-state response is significant, the transient part of eqn. (10) is disregarded. The steady-state response $C_{1}(0, t)$ is

$C_{1}(0, t)=C_{0}+\frac{K}{\omega} \sin \omega t-\frac{K}{\omega} \exp \left[-\left(\frac{\omega}{2 D}\right)^{1 / 2} l\right] \sin \left[\omega t-\left(\frac{\omega}{2 D}\right)^{1 / 2} l\right]$

Equation (12) can be rearranged as

$C_{1}(0, t)=C_{0}+A \cos (\omega t+\phi)$

where

$A=\frac{K}{\omega}\left\{1-2 \exp \left[-\left(\frac{\omega}{2 D}\right)^{1 / 2} l\right] \cos \left(\frac{\omega}{2 D}\right)^{1 / 2} l+\exp \left[-2\left(\frac{\omega}{2 D}\right)^{1 / 2} l\right]\right\}^{1 / 2}$

$\phi=-\frac{\pi}{2}+\tan ^{-1}\left\{\frac{\exp \left[-\left(\frac{\omega}{2 D}\right)^{1 / 2} l\right] \sin \left[\left(\frac{\omega}{2 D}\right)^{1 / 2} l\right]}{1-\exp \left[-\left(\frac{\omega}{2 D}\right)^{1 / 2} l\right] \cos \left[\left(\frac{\omega}{2 D}\right)^{1 / 2} l\right]}\right\}$

The response is sinusoidal with the same frequency as the applied current. Its amplitude and phase shift are dependent on the frequency of the applied current, the diffusion coefficients of the active species as well as the thickness of the porous actuator. It must be noted that the phase shift $\phi$ of the concentration change given in eqn. (13) is defined with respect to the titrant production rate. If the phase shift is measured with respect to the applied current, $i$, the initial phase shift $\theta$ has to be included as indicated in eqn. (2).

The amplitude and phase shift of the change in concentration with respect to the alternatively generated titrant described by eqn. (14) are plotted in Fig. 4 as

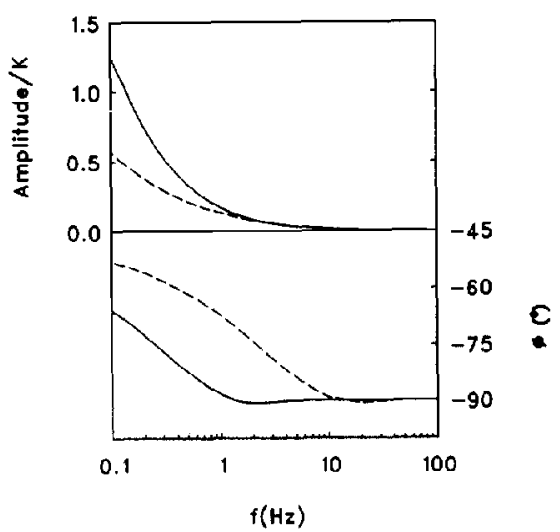

Fig. 4. Normalized amplitude and phase shift of the change in concentration as a function of frequency according to eqn. (14). Dashed lines are calculated by substituting the diffusion coefficient of protons and solid lines are for acetic acid. a function of the frequency. The thickness of the actuator is assumed to be $50 \mu \mathrm{m}$ for the calculation in this plot. The diffusion coefficients are those of protons (dashed lines) and acetic acid molecules (solid lines). The graph shows that as the frequency increases, the amplitude decreases and the phase shift approaches $-90^{\circ}$. At very low frequency the phase shift will be $-45^{\circ}$.

It should be mentioned here that a part of the total applied current, $i_{\text {toral, }}$, will be used for the double-layer charging. The amplitude of the double-layer charging current, $i_{\mathrm{dl}}$, is a function of the frequency of the applied current, so the amplitude of the faradaic current, $i_{\mathrm{g}}$, will also be a function of the frequency of the applied current $i_{\text {total }}$ because $i_{\text {total }}=i_{\mathrm{f}}+i_{\mathrm{dl}}$. The titrant production rate, as defined in eqn. (3), is in this instance not only proportional to the amplitude of the applied current, but also to the frequency. As the frequency increases, the double-layer charging becomes more important. Consequently, the amplitude of the faradaic current decreases and the amount of generated species will be less. As a consequence, the titrant production rate decreases. At higher frequencies, the production rate will even practically drop to zero, because the faradaic process hardly proceeds at high frequencies. Due to the effect of the double-layer charging, the amplitude of the response with respect to the applied current will decrease with increasing frequency even faster than expected from eqn. (14). The phase shift will practically go to zero instead of $-90^{\circ}$ at high frequency.

\section{Buffer-capacity-dependent system responses}

Considering a buffer system consisting of a weak acid, $\mathrm{HA} \rightleftharpoons \mathrm{H}^{+}+\mathrm{A}^{-}$, the buffer capacity $\beta$, defined as 
a small amount of base $\mathrm{d}[\mathrm{B}]$ needed to change the $\mathrm{pH}$ by $\mathrm{dpH}$, is given by [9]

$\beta=\frac{\mathrm{d}[\mathrm{B}]}{\mathrm{dpH}}=2.3\left\{\frac{K_{\mathrm{a}} C_{0}\left[\mathrm{H}^{+}\right]}{\left(K_{\mathrm{a}}+\left[\mathrm{H}^{+}\right]\right)^{2}}+\left[\mathrm{H}^{+}\right]+\frac{K_{\mathrm{w}}}{\left[\mathrm{H}^{+}\right]}\right\}$

where $K_{\mathrm{w}}$ is the ion-product of water, $K_{\mathrm{a}}$ is the dissociation constant of the acid HA and $C_{0}$ is the initial bulk concentration of the acid, i.e., $[\mathrm{HA}]+\left[\mathrm{A}^{-}\right]$.

For the addition of a small amount of titrant $\Delta[B]$, the change of the bulk concentration is sufficiently small. The corresponding $\mathrm{pH}$ change, $\Delta \mathrm{pH}$, is

$$
\begin{aligned}
\Delta \mathrm{pH} & \cong \frac{\mathrm{dpH}}{\mathrm{d}[\mathrm{B}]} \Delta[\mathrm{B}] \\
& =\frac{\Delta[\mathrm{B}]}{2.3\left\{\frac{K_{\mathrm{a}} C_{0}\left[\mathrm{H}^{+}\right]}{\left(K_{\mathrm{a}}+\left[\mathrm{H}^{+}\right]\right)^{2}}+\left[\mathrm{H}^{+}\right]+\frac{K_{\mathrm{w}}}{\left[\mathrm{H}^{+}\right]}\right\}}
\end{aligned}
$$

If the addition of titrant is coulometrically generated at the porous actuator, the effective change of titrant $\Delta[B]$ should be taken as equivalent to the associated change of the concentration of the species that will be detected by the ISFET at $x=0$, i.e., $\Delta[\mathrm{B}]=\Delta C_{1}(0, t)$ (see eqn. (13)) and the $C_{0}$ in the denominator of eqn. (16) should be replaced by $C_{1}(0, t)$. The response to this $\mathrm{pH}$ change $\Delta \mathrm{pH}$ at the gate of the ISFET is obtained by incorporating eqn. (13) in (16):

$$
\begin{aligned}
\Delta \mathrm{pH}(0, t)_{\mid \mathrm{ISFET}} & \cong \frac{\mathrm{dpH}}{\mathrm{d}[\mathrm{B}]} \Delta C_{1}(0, t) \\
& =\frac{A \cos (\omega t+\phi)}{2.3\left\{\frac{K_{\mathrm{a}} C_{1}(0, t)\left[\mathrm{H}^{+}\right]}{\left(K_{\mathrm{a}}+\left[\mathrm{H}^{+}\right]\right)^{2}}+\left[\mathrm{H}^{+}\right]+\frac{K_{\mathrm{w}}}{\left[\mathrm{H}^{+}\right]}\right\}}
\end{aligned}
$$

where $A$ and $\phi$ are given by eqn. (14). The concentration $C_{1}(0, t)$ can be considered as constant and equal to the bulk concentration $C_{0}$ for a small titrant perturbation. If the concentration $C_{0}$ is relatively low compared to the titrant, it will be given by eqn. (13) instead.

Equation (17) shows that the response of the ISFET with respect to a coulometrically generated titrant perturbation is inversely proportional to the buffer capacity of the analyte, provided the titrant perturbation is small. It indicates that the buffer capacity can be determined. It can be seen that this dynamic way of measuring differs from the conventional derivative titration in that the titrant is locally generated by coulometry and the associated local $\mathrm{pH}$ changes are rapidly detected by the ISFET. The advantage is that the measurement can be carried out very rapidly without disturbing the bulk solution. However, it should be noticed that the response is also a function of the frequency of the applied current and the geometric dimension of the actuator as well as the mobility of the species (see eqn. (14)). The former two variables will not cause problems for a measurement, because the frequency can be optimized and fixed during the measurement. The geometric dimensional parameters are also constant for a particular device design. The dependence of the response on the mobility of the ions will, however, influence the accuracy of the measurement. As has been noticed before $[5,7]$, the protons have a strong influence on the diffusion due to their high mobility. It can be expected that the response of the system during the measurement in an acidic solution will be lower than in a basic solution. One way to minimize this effect is to increase the frequency $\omega$ of the alternating titrant or the thickness $l$ of the actuator according to eqn. (14). If the condition $(\omega / 2 D)^{1 / 2} l>3$ is satisficd, the valuc of the exponential term $\exp \left[-(\omega / 2 D)^{1 / 2} l\right]$ in eqn. (14) will be less than 0.05 and it can be omitted. The amplitude of change in concentration can be considered as independent of the diffusion coefficient of the species. However, the increase of $l$ is limited by the available technology.

Another difference of this measurement from a conventional derivative titration is that a phase shift with respect to the titrant production rate or to the applied current is predicted and can be measured. In principle, this has no negative effect on the measurement but can even provide extra information.

It should be pointed out that in this derived model only mass transport is considered. If the dissociation and association of protons or hydroxyl ions are kinetically slow, which might occur in some other applications, the description of the response should be modified. However, this topic is beyond the scope of this paper and will not be considered here.

Equation (17) can be simplified in some special cases. For a strong acid of concentration $C_{0}$, the buffer capacity can be simplified from eqn. (17) to

$$
\begin{aligned}
\Delta \mathrm{pH}(0, t)_{\mid \text {ISFET }} & \cong \frac{\mathrm{dpH}}{\mathrm{d}[\mathrm{B}]} \Delta C_{1}(0, t) \\
& =\frac{\mathrm{A} \cos (\omega t+\phi)}{2.3\left[\mathrm{H}^{+}\right]} \cong \frac{A \cos (\omega t+\phi)}{2.3 C_{0}}
\end{aligned}
$$

It can be seen that the change of $\mathrm{pH}$ is inversely proportional to the bulk concentration of the acid, which means that the buffer capacity is proportional to the concentration of a strong acid.

For a weak acid of concentration $C_{0}$, with the dissociation constant $K_{\mathrm{a}}$ so small that the condition $\left[\mathrm{H}^{+}\right] \gg K_{\mathrm{a}}$ is satisfied, eqn. (17) is simplified for $11>\mathrm{pH}>3$ to 


$$
\begin{aligned}
\Delta \mathrm{pH}(0, t)_{\mid \mathrm{ISFET}} & \cong \frac{\mathrm{dpH}}{\mathrm{d}[\mathrm{B}]} \Delta C_{1}(0, t) \\
& \cong \frac{\left(K_{\mathrm{a}}+\left[\mathrm{H}^{+}\right]\right)^{2}}{2.3 K_{\mathrm{a}} C_{0}\left[\mathrm{H}^{+}\right]} A \cos (\omega t+\phi) \\
& \cong \frac{A \cos (\omega t+\phi)}{2.3\left(K_{\mathrm{a}} C_{0}\right)^{1 / 2}}
\end{aligned}
$$

The change of $\mathrm{pH}$ is inversely proportional to the square root of the product of $K_{\mathrm{a}}$ and the concentration of the weak acid.

\section{Experimental}

\section{Sensor-actuator device}

The procedure to make the coulometric sensor-actuator device that has been described before $[6$, 7] is shown in Fig. 5. A flat ISFET [10] is used as the $\mathrm{pH}$ sensor with an additional thick layer of $\mathrm{Ta}_{2} \mathrm{O}_{5}$ of about $150 \mathrm{~nm}$ as a barrier to prevent gold from migrating into the silicon. The starting layer of the actuator was a $0.5 \mu \mathrm{m}$ layer of gold deposited on the ISFET chip by thermal evaporation (Fig. 5(a)). After patterning by photolithography, an $\mathrm{SiO}_{2}$ layer of approximately $3 \mu \mathrm{m}$ thickness was deposited by PECVD (plasma-enhanced chemical vapour deposition) on the top of the gate as the sacrificial layer (Fig. 5(b)). Then, a layer of gold thick-film paste (DuPont 9910) was applied (Fig. 5(c)). After drying, the thick-film paste was sintered at $600^{\circ} \mathrm{C}$ for one hour in a nitrogen atmosphere. The etching of the sacrificial layer and the glass compound in the thick-film paste was carried out in buffered HF for one and a half hours under stirring conditions (Fig. 5(d)). A typical thickness of the obtained porous actuator is $\approx 100 \mu \mathrm{m}$. The chip was finally mounted on a printed circuit board and encapsulated by epoxy.

\section{Set-up}

The buffer capacity measuring set-up is shown in Fig. 6. Since the current source is not floating, the grounded counter electrode works as a reference electrode as well. In this case, the current at low frequency will cause a certain polarization in spite of the very large area of the counter electrode. This polarization potential of the counter electrode will be superposed on the output of the ISFET amplifier and interfere with the measurement. Therefore, an additional saturated calomel electrode is used to measure the polarization potential separately and the signal is sent to the lock-in amplifier for subtraction. The measured current and voltage are presented in effective (root mean square or r.m.s.) values.

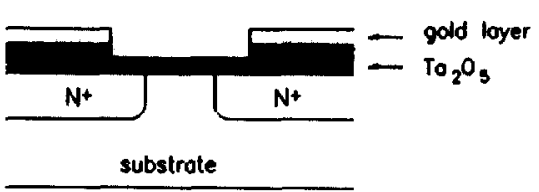

(a)

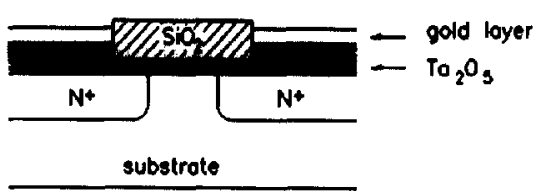

(b)

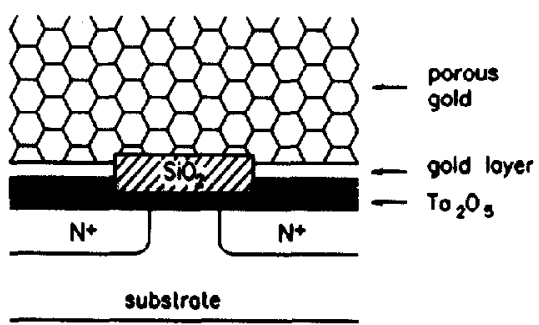

(c)

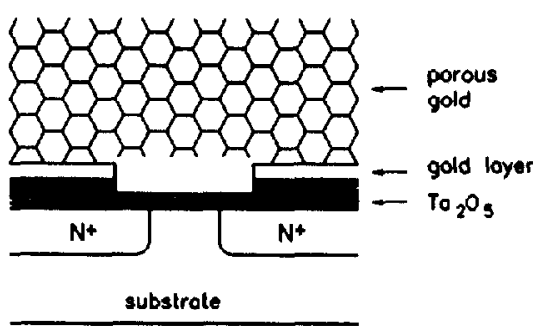

(d)

Fig. 5. Procedure to make the coulometric sensor-actuator device: (a) flat ISFET with a thin layer of evaporated gold around the gate; (b) deposition of a sacrificial layer of $\mathrm{SiO}_{2}$ by PECVD; (c) deposition of a layer of porous gold; (d) etching of the sacrificial layer.

\section{Measurement protocol}

The measurement was carried out in a glass vessel at room temperature. Before each measurement nitrogen was bubbled through the solution for $15 \mathrm{~min}$ to get rid of the influence of carbon dioxide. The vessel was purged with nitrogen throughout the measurement. The measurement started from a high $\mathrm{pH}$ to decrease further the influence of carbon dioxide. The $\mathrm{pH}$ was changed by adding $1.0 \mathrm{M}$ nitric acid or $1.0 \mathrm{M}$ potassium hydroxide and was monitored by a $\mathrm{pH}$ meter with a glass electrode (Radiometer). The current source was built by using the voltage output of a signal generator in a series connection with a $1 \mathrm{M} \Omega$ resistor. The counter electrode was a large copper strip with plated gold on 


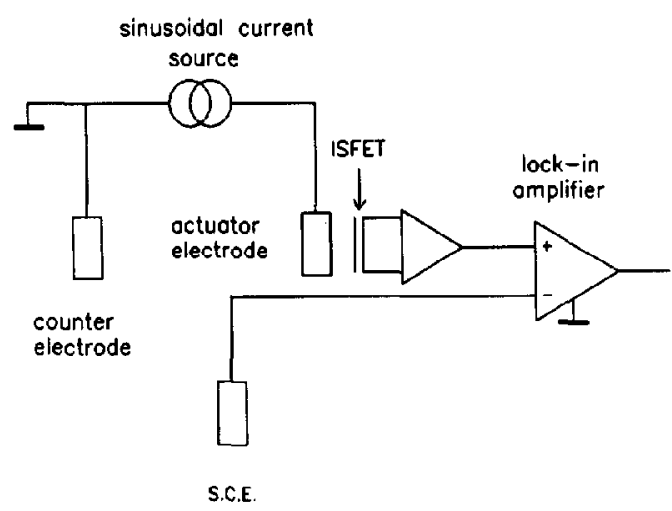

Fig. 6. Set-up for buffer capacity measurement.

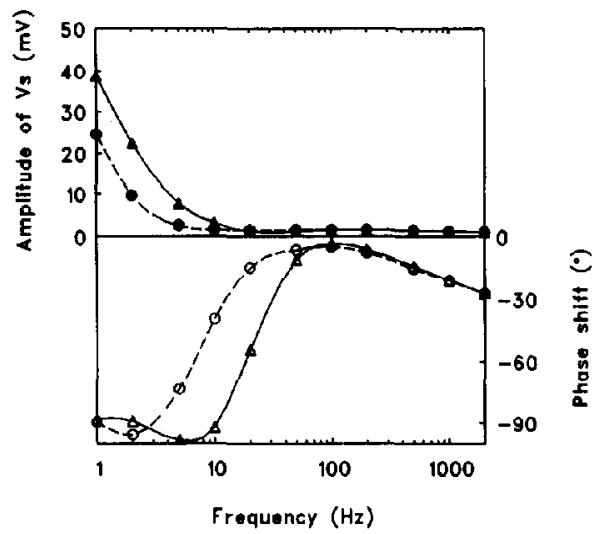

Fig. 7. Output (r.m.s.) of ISFET amplifier as a function of the frequency of the applied current. $I_{\text {eff }}=10 \mu \mathrm{A} ;\left[\mathrm{KNO}_{3}\right]=1 \mathrm{M} ; \Delta$, $\Delta,[\mathrm{HAc}]=2 \mathrm{mM} ; 0, \mathrm{O},\left[\mathrm{HNO}_{3}\right]=2 \mathrm{mM}$.

its surface. The lock-in amplifier is a PAR ${ }^{\mathrm{TM}}$ model $129 \mathrm{~A}$.

\section{Results and discussion}

The response of the system to the sinusoidal current was recorded. The output of the ISFET amplifier that reflects the small change of $\mathrm{pH}, \Delta \mathrm{pH}$, was found to be sinusoidal as anticipated. The amplitude of the output $V_{\mathrm{s}}$ and the phase shift with respect to the applied current were measured by a lock-in amplifier. The response of the system in nitric acid (circles) and in acetic acid (triangles) as a function of the frequency is shown in Fig. 7. The applied current, $I_{\text {effi }}$ is $10 \mu \mathrm{A}$.

From this experiment it is concluded that at low frequency $(f<5 \mathrm{~Hz})$, a sinusoidal current applied to the actuator will result in a small local sinusoidal change of $\mathrm{pH}$. The response of the ISFET to this $\mathrm{pH}$ change is a function of the frequency of the current. The amplitude of the response of the ISFET decreases as the frequency increases, because of the decrease of the faradaic current with the frequency as well as the limitation of the diffusion rate of the species at high frequency (see eqn. (14)).

As the frequency increases from 5 to $10 \mathrm{~Hz}$, the amplitude of the ISFET amplifier output decreases to around zero while the phase shift changes rapidly from $\approx-90$ to $\approx 0^{\circ}$. This indicates that at high frequencies no protons or hydroxyl ions are generated and the current is used only for the double-layer charging. The residual small output signal is only a measure of the solution resistance. If this result is compared with Fig. 4 , one can conclude that the amplitude of the output approximates the theoretically predicted curve. However, the measured phase shift shows a difference with respect to Fig. 4 for the frequencies above $5 \mathrm{~Hz}$. The reason is that the faradaic current drops to zero and the solution resistance will give no phase shift with respect to the current. Another difference of this result from the theoretical prediction as given by eqn. (14) is caused by the effect of the initial phase shift $\theta$ (see eqn. (2)), because the phase shift $\phi$ described by eqn. (14) is with respect to the titrant production rate $\rho_{\mathrm{f}}$ (eqn. (3)), but the measured phase is with respect to the applied current $i$ (eqn. (2)). The measured phase will shift more than predicted by eqn. (14) if this initial phase shift for low frequencies is taken into account, as can be seen for $f<5 \mathrm{~Hz}$ in Fig. 7. The important conclusion from this result is that the sinusoidal generation of titrant is possible, but is limited to frequencies below $10 \mathrm{~Hz}$.

The measurement result also shows that the amplitude of the ISFET amplifier output in acetic acid is higher than in nitric acid. This proves that the response is also a function of the buffer capacity of the solution, since a fully dissociated acid has a higher buffer capacity. However, the amplitude differences of the output for the different acids are not exactly the same as calculated with eqns. (18) and (19). The reason is that these equations are simplified by assuming a constant concentration of the buffer solution and the concentration of titrant, d[B], is assumed to be infinitely small. In practice the change of titrant $\Delta[B]$ is relatively large if the buffer capacity is low and in this case the titrant itself will also function as a buffer.

The buffering property of acetic acid at different $\mathrm{pH}$ values was measured and the result is shown in Fig. 8 (triangles with solid line in upper part of the Figure). A frequency of $1 \mathrm{~Hz}$ was chosen in order to obtain a relatively high output. The measurement results are presented as the reciprocal of the ISFET amplifier potential $V_{s}$. This reciprocal expression of the results directly reflects the buffer capacity as a function of $\mathrm{pH}$. At $\mathrm{pH}=\mathrm{p} K_{\mathrm{a}}$, a maximum buffer capacity can be found from the experimental results. It is in fairly good agreement with the theoretical description. For the 


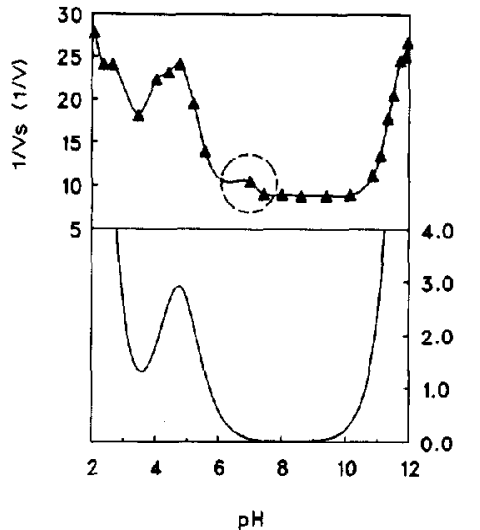

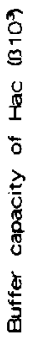

Fig. 8. Buffer capacity of $5 \mathrm{mM}$ acetic acid as a function of $\mathrm{pH}$. $\left[\mathrm{KNO}_{3}\right]=0.1 \mathrm{M}, l_{\text {eff }}=10 \mu \mathrm{A}, f=1 \mathrm{~Hz}$. Upper marks and fitting line are from measurement, lower curve is calculated.

comparison, the theoretical value according to eqn. (15) was calculated and is also shown in Fig. 8 (lower part of the Figure). It can be seen that the measurement curve is quite similar to that of the theoretical calculation. However, a small terrace has been found in the experimental curve below $\mathrm{pH}=7$, as is encircled in Fig. 8. This effect is due to the high mobility of the protons, as has been mentioned before. It can also be explained by referring to Fig. 4 or eqn. (14) in that the output of the ISFET response is also a function of the diffusion coefficients of the species. In a buffered region ( $\mathrm{pH}=\mathrm{pK} \pm 1)$, the dominant diffusion species are the acid molecules and therefore their diffusion coefficient has to be substituted into eqn. (14) for calculation. In this experiment the buffer region of acetic acid is around $\mathrm{pH}=\mathrm{p} K_{\mathrm{a}}=4.74$. Beyond this buffer range the diffusion coefficient of the protons should be substituted into eqn. (14) for $\mathrm{pH}<7$ (except $\mathrm{pH}=\mathrm{p} K \pm 1)$ and that of the hydroxyl ions has to be substituted into eqn. (14) for $\mathrm{pH}>7$. This will result in an amplitude difference above and below $\mathrm{pH} 7$. These experimental results and calculations mean that the mobility of protons or hydroxyl ions will also influence the measurement, which causes a deviation in these dynamic measurement results from that measured in a static way, such as a volumetric titration. This diffusion effect functions as a kind of interference for the dynamic buffer capacity measurement. The possible ways to reduce the effect are either to apply a thicker actuator or to increase the frequency of the applied current during operation according to eqn. (14). However, a thick actuator is technologically difficult to make and the increase in frequency is restricted by the effect of the double-layer charging, as has been discussed before. Applied currents at frequencies above $10 \mathrm{~Hz}$ will not effect a coulometric generation of titrant. Moreover, the smaller perturbation of titrant by a high-

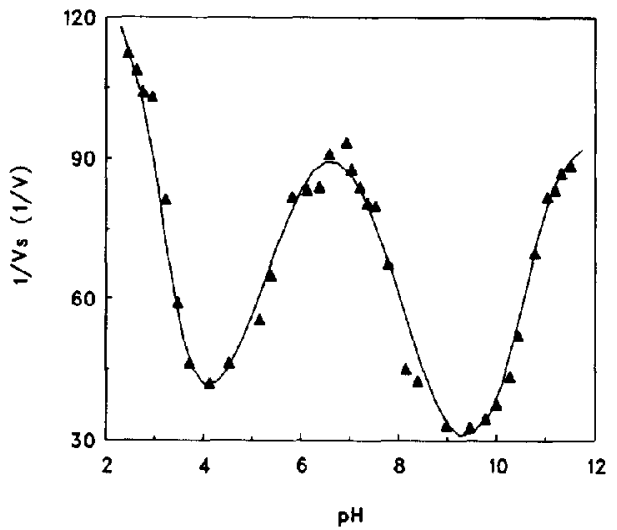

Fig. 9. Buffer capacity measurement of $10 \mathrm{mM}$ phosphoric acid as a function of $\mathrm{pH} .\left[\mathrm{KNO}_{3}\right]=0.1 \mathrm{M}, I_{\mathrm{eff}}=10 \mu \mathrm{A}, f=1 \mathrm{~Hz}$.

frequency current will result in a smaller $\mathrm{pH}$ change, causing a very small output signal of the ISFET, thereby also decreasing the measurement resolution.

Besides the measurement of the buffer capacity of the monobasic acid solution, phosphoric acid was also measured at different $\mathrm{pH}$ values and the results are presented in Fig. 9 as the reciprocal of the ISFET recording. The theoretical curve is not presented because the calculation is rather cumbersome. However, the available knowledge from earlier studies is adequate for evaluating the experimental results [11]. It is known that the maximum buffer capacity appears at the $\mathrm{p} K$ values of a polybasic acid. It is therefore expected that the buffer capacity curve of phosphoric acid will have three peaks at $\mathrm{p} K_{1}=2.15, \mathrm{p} K_{2}=7.2, \mathrm{p} K_{3}=12.32$. The peak around $\mathrm{pH}=7.2$ was distinctively present in the measurement, whereas the other two peaks are not seen because they are within the solvent buffering region, i.e., strong acid or base buffering region. The high concentration of protons or hydroxyl ions below $\mathrm{pH}=3$ and above $\mathrm{pH}=11$ will show a high buffer capacity, which makes it difficult to distinguish the other two peaks from this buffer capacity curve of phosphoric acid. Similar to the measurement in acetic acid, the buffer capacity is clearly higher in the acidic region than in the basic region, as explained above. Nevertheless, the results from measurements are in good agreement with the theoretical buffer capacity of an analyte.

\section{Conclusions}

In this paper, a dynamic way of sensing the buffer capacity by means of a coulometric sensor-actuator system with a gate-covering porous actuator has been proposed. The buffer capacity is measured by a small sinusoidal change in the concentration of titrant, which 
is coulometrically generated by applying a sinusoidal current to the porous actuator. The experimental results have shown that the frequency range for an effective generation of sinusoidal perturbation of titrant is below $10 \mathrm{~Hz}$. The amount of titrant generated decreases with the increase of the frequency of the applied current, because of double-layer charging interference. The detected change in $\mathrm{pH}$ by the ISFET is found to be dependent on the buffer capacity of the analyte. Because the diffusion effect also contributes to the measurement, the mobility of protons, being higher than that of hydroxyl ions, will cause the measured $\mathrm{pH}$ change to be smaller in the acidic region than in the basic region. Consequently, our results show. a small deviation from those obtained with static measurement methods, and this should be taken into account in interpreting the dynamic measurement results.

\section{References}

1 W. Olthuis, B.H. van der Schoot, F. Chavez and P. Bergveld, A dipstick sensor for coulometric acid-base titration. Sensors and Actuators, 17 (1989) 279-283.

2 B.H. van der Schoot and P. Bergveld, Coulometric sensors: ISFETs with a coulometric $\mathrm{pH}$-actuator for long-term use without calibration, Proc. 4th Int. Conf. Solid-State Sensors and Actuators (Transducers' 87), Tokyo, Japan, June 2-5, 1988, pp. 719-722.

3 B.H. van der Schoot and P. Bergveld, Coulometric sensors, the applications of a sensor-actuator system for long-term use in chemical sensing, Sensors and Actuators, 13 (1988) 251-262.

4 B.H. van der Schoot and P. Bergveld, The pH-static enzyme sensor, an ISFET-based enzyme sensor, insensitive to the buffer capacity of the sample, Anal. Chim. Acta., 199 (1987) $157-160$.

5 W. Olthuis, J. Luo, B.H. van der Schoot, P. Bergveld, M Bos and W.E. van der Linden, Modelling of non-steady-state concentration profiles at ISFET-based coulometric sensoractuator systems, Anal. Chim. Acta., 229 (1990) 71-81.

6 J. Luo, W. Olthuis, P. Bergveld, M. Bos and W.E. van der Linden, A porous gold actuator for the ISFET-based coulometric sensors-actuator systems, Proc. 6th Int. Conf. Solidstate Sensors and Actuators (Transducers' 91), San Francisco, CA, USA, June 24-28, 1991, pp. 229-232.

7 J. Luo, W. Olthuis, P. Bergveld, M. Bos and W.E. van der Linden, Modelling of coulometric sensor-actuator systems based on an ISFET with a porous actuator covering the gate, Anal. Chim. Acta, 274 (1993) 7-23.

8 H.S. Carslaw and J.C. Jaeger, Conduction of Heat in Solids, Oxford University Press, London, 1959, p. 317.

9 D.D. Perrin and B. Dempsey, Buffers for $\mathrm{pH}$ and Metal Ion Control, Chapman and Hall, London, 1974, p. 11.

$10 \mathrm{~J}$. Luo, Employment of a porous gold actuator in ISFETbased coulometric sensor-actuator systems, with application to protein characterization, Dissertation, University of Twente, 1993, Ch. 4, p. 87.

11 G.H. Jeffery, J. Bassett, J. Mendham and R.C. Denney, Vogel's Textbook of Quantitative Chemical Analysis, Longman Scientific \& Technical, Harlow, 1989, p. 276.

\section{Biographies}

Jiang Luo was born in Wuhan, China, on September 14,1956 . He received the B.S. degree in electrical engineering from Huazhong (Central China) University of Science and Technology in 1982 and the M.S. degree in biomedical engineering from Tongii Medical University in 1987. In September 1988, he joined the Biosensor Technology Group in the Faculty of Electrical Engineering, University of Twente, Enschede, The Netherlands and started his Ph.D. research work on ISFETbased coulometric sensor-actuator systems.

Wouter Olthuis was born in Apeldoorn, The Netherlands, on October 23, 1960. He received the M.S. degree in electrical engineering from the University of Twente, Enschede, The Netherlands in 1986, and the Ph.D. degrce from the Biomedical Engineering Division of the Faculty of Electrical Engineering, University of Twente, in 1990. Currently he is working as an assistant professor in the Biosensor Technology Group of the University of Twente.

Piet Bergueld was born in Oosterwolde, The Netherlands, on January 26,1940 . He received the M.S. degree in electrical engineering from the University of Eindhoven, The Netherlands, in 1965 and the Ph.D. degree from the University of Twente, The Netherlands, in 1973. The subject of his dissertation was the development of ISFETs and related devices. Since 1965 he has been a member of the Biomedical Engineering Division of the Faculty of Electrical Engineering at the University of Twente and was appointed as full professor in Biosensor Technology. $\mathrm{He}$ is one of the project leaders in the MESA Research Institute. His research subjects still concern the further development of ISFETs and hiosensors based on ISFET technology.

Martinus Bos received his M.S. degree in chemical technology at the Technical University of Delft, The Netherlands, in 1965. He received his Ph.D. degree in 1972 from the University of Twente for his work in analytical chemistry. $\mathrm{He}$ is a staff member since 1972 of the Chemical Analysis Group of the Faculty of Chemical Technology at the University of Twente. His research areas are electro-analysis and chemometrics.

Willem E. van der Linden studied at the University of Amsterdam (The Netherlands) and received his Ph.D. in analytical chemistry in 1966 . He stayed at this university until 1980, when he was appointed as a professor in chemical analysis at the University of Twente, Enschede. His fields of interest are electroanalytical chemistry and flow analysis, in particular flow-injection analysis, and their use in process analytical chemistry. $\mathrm{He}$ is one of the editors of Analytica Chimica Acta. 